\title{
Midwifery continuity: the use of social media
}

\author{
Introduction
}

Pregnancy, birth and early motherhood are times of significant change and transformation for women (Darvill et al., 2010). Effective midwifery care and support provided for women through this transformational period is not only beneficial for the woman, but their children and wider communities (National Health Service England (NHS), 2016). Foundations for long-term health outcomes are established during pregnancy and early years of life which can significantly influence health outcomes for children and adults as such it is a critical time in terms of maternal, child and family health and wellbeing (Sandall et al., 2016; Marmot et al., 2010; Shribman \& Billingham, 2009).

A fundamental aspect of contemporary midwifery care is continuity of care and relationship building to provide effective support for women through pregnancy, birth and early motherhood. It is essential that relationships are formed which can facilitate trust, enable personalised care and bridge gaps and discontinuities in support, to achieve positive physical and psychological outcomes for women (Sandall et al., 2016a; Sandall et al., 2016). Continuity models of midwifery care acknowledge the importance of relationships and meaningful connections developing between women and midwives (Sandall et al., 2016a; Sandall et al., 2016; McLachlan et al., 2016, Tracy et al., 2013).

In a broad health context continuity is associated with improvements in care and is achieved when three fundamental elements are realised: relational continuity through sustained therapeutic relationships with known health professionals; management continuity with seamless communication about and between women, health professionals and health organisations; informational continuity through timely access to relevant information (Heaton, Corden \& Parker, 2012; Freeman et al., 2007). Relational continuity has the greatest influence on experience and outcomes, and cannot be substituted by information and management continuity (Sandall et al., 2016a; Guthrie et al., 2008).

Continuity in maternity care is achieved when a known midwife follows a woman through her childbirth experiences regardless of the complexity of the pregnancy, and irrespective of the place where care is provided. This type of relationship based care provides multiple benefits for women including improved health outcomes, a greater sense of satisfaction with their childbirth experience and a greater sense of agency and control (Sandall et al., 2016; McLachlan et al., 2016; Walsh \& Devane, 2012).

Continuity models of midwifery care have been advocated since 1993 and relationship based, personalised care forms the basis of current maternity policy in the UK (Department of Health (DH), 1993; DH, 2010; NHS England, 2016). These models have been largely hospital or community based, and can provide continuity to low and high risk women. The most common continuity models are achieved through case-loading or team midwifery (Sandall et al., 2016). Case-loading midwifery usually consists of a midwife providing care to a group of women and handing over to a midwifery partner when unavailable. Team midwifery is provided 
by a team of midwives, one of whom will provide most care but women may meet other members of the team (Sandall et al., 2016a). Both models are underpinned by the concept of a named and known midwife providing the majority of care and both models have been successfully implemented in maternity services with no significant differences in outcomes (Sandall et al., 2016a).

Despite being cost neutral with compelling evidence supporting extensive implementation, most women still do not receive continuity of midwifery care (NHS England, 2016; Sandall et al., 2016a; McLachlan et al., 2016; Kenny et al., 2015). The most common reasons for this are attributed to resource constraints, modern working practices, and the current trend to centralise NHS maternity services. As a result large teams of midwives provide fragmented care to women inhibiting the development of trusting relationships and denying women their rights to safe and high quality maternity care (McLachlan et al., 2016; Renfrew et al., 2014; Page \& McCandlish, 2006).

The underpinning premise of relational continuity is based on the sustained and ongoing midwife mother-relationship. Continuity models need to be scalable and sustainable for all women to receive equitable benefit. Current continuity models have not met service provider conditions for widespread implementation. This paper reports initial qualitative findings, from a larger mixed methods research study, that suggest relational continuity in midwifery care can, for some women, be achieved through online contact, communication and support accessed through a social media platform. This model has the potential to be scalable and sustainable. Given the recent publication 'Relationships: the pathway to safe, high-quality maternity care (Sandall et al., 2016) calling for continuity models of care the authors believed it was important to release such findings early (prior to the whole study report) to expose new ideas and approaches for maternity care that could be considered.

\section{Methods}

\section{Study design}

This paper reports the early findings from a larger mixed methods study exploring the use of online communities within a midwifery context, to increase knowledge and social learning to promote health. The focus of this paper is on the embedded qualitative study data, and the experiences of pregnant women $(n=31)$ and midwife moderators $(n=4)$ who were involved in online Facebook groups throughout their pregnancy, gathered using repeated longitudinal focus groups $(n=8)$ and individual interviews $(n=28)$.

\section{Setting, sample and recruitment}

Two large NHS Foundation Trusts agreed to support the study and provided access to service users referred to the maternity services. All pregnant women attending for a baseline dating scan or booking appointment (between 6-12 weeks approximately) were potential participants and were approached by the attending midwife $(n=106$ total 64/42) and provided with study information material. 
Of these 106, $72(48 / 24)$ completed an expression of interest form and were approached by the female researcher. Two women were no longer pregnant therefore not eligible for the study and 28 women failed to respond to the researcher contact, despite two follow up prompts. Therefore, out of 72 women contacted and invited to join the research study, 31 agreed (43\% response rate) although only $30 \%$ of the target population (Table 1).

Table 1 - recruitment numbers

The inclusion criteria for the study was restricted to pregnant, English speaking women aged 16 years and over, without a serious mental health condition. The rationale was pragmatic, based on the need for midwives, without additional specialist skills, to be able to moderate the site and communicate effectively with all participants. The majority of women who refused to take part did so because of the demanding schedule of the research (to attend four focus groups and one interview) during their pregnancy.

Four midwives were recruited, two from each Trust and seconded to the research project for the duration of the study (35 weeks) they also participated as additional study participants. Demographic information about the midwives is detailed in Table 1a. The midwives went through a competitive recruitment process and were selected for their enthusiasm about using social media as an adjunct to routine midwifery care and for having a visible social media presence.

Table 2 - Demographic details about the midwife moderators (Facewives).

\section{Online Facebook Groups}

Facebook provided a platform for the creation of a virtual meeting space for each group. Facebook was selected because it is the largest social media platform most commonly used by women, and its' highest use is reported amongst women aged 18-29 years (Duggan \& Smith, 2013; Fox, 2011). Specialist equipment and training are not required when using Facebook, it can be easily accessed from different devices connected to the internet (Bacigalupe, 2011).

A secret Facebook group was set up by the respective midwife moderators for each Trust. Facebook has three levels of privacy setting and secret is the highest. The secret privacy setting meant that the group was open and visible to invited members only, secret groups are not google indexed or searchable within or outside Facebook. This was an important feature which safeguarded the privacy and confidentiality of members from the outset. A set of expectations for conduct on the site was derived and posted for participants use, guided by the social media advice from the Nursing and Midwifery Council (NMC) (2015) and experience from another project at the host institution (Vasilica, 2015). The midwives were professionally bound by the Midwives Rules and Standards (NMC, 2012) and the Code of Conduct (NMC, 2008) and Ethical approval from National Research Ethics Service (NRES) and the host institution was obtained. 
The remit of the midwife moderator was to be available to answer queries and verify information online every day, several times a day. Each midwife was paid for 7.5 hours per week to moderate the group site, with a commitment to ensure a midwifery presence on the site with no gaps of over eight hours (i.e. overnight). The midwives went through a competitive recruitment process and were selected for their enthusiasm about using social media as an adjunct to routine midwifery care and for having a visible social media presence. The midwife moderators were trained and guided in engagement techniques by the research team and by a researcher with expertise in social media engagement. Each group used these differently, influencing engagement through posting or sharing information, starting conversations/discussions, clarifying answers to questions posed. Conversations were usually about pregnancy related issues, but part of the engagement involved both Facewives and Facemums sharing personal aspects about non pregnancy related issues. The private message function was available for sensitive communication. A researcher monitored each Facebook site and made suggestions to the moderators about potential content for posts, and strategies to engage members and generate activity. Whilst there were no breaches of confidentiality, a governance structure was established to ensure escalation of any breach to the principal researcher.

The online group and participants took the title 'Facemums' with the midwife moderators being referred to as Facewives. Each group/participant/moderator was further recognised by the name of the Trust they were affiliated to (FMB/FMC).

\section{Data Collection \& Analysis}

Online activity data was created by the participants and midwife moderators for the duration of the project (35 weeks). Focus groups were conducted at approximately 10-12 week intervals. The focus groups were conducted by the same researcher using the same interview schedule. Four were conducted on line and four were in person. After each face to face focus group was completed, the audio recordings were transcribed by the primary researcher and checked by the co-researcher for accuracy. The online focus group data were saved in a word document format. Individual face to face interviews were conducted with participants within six weeks of their birth experience. Interviews were also conducted with each of the midwife moderators. Twenty eight individual interviews were conducted, (15 women +2 midwives / 9 women +2 midwives). After each interview was completed, the audio recordings were transcribed and checked for accuracy.

A thematic analysis using a priori themes was undertaken. This involved coding data which evidenced relational, informational and management continuity across the entire dataset (28 interviews and 8 focus groups). The analysis was undertaken broadly following the six stages described by Braun and Clarke (2013): familiarisation and data transcription, initial coding and then searching for and reviewing the identified themes. The primary investigator analysed the data using Braun and Clarke's six stage model and the co-researcher checked and confirmed the identified themes. 


\section{Results}

A total of 31 women, and 4 midwives, participated in the study. The demographic data of the participants (on recruitment) regarding their age, parity, education and employment status is detailed in table 1 and shows their varied backgrounds and reflects the ethnicity demographic of the Trusts where the research was based. The educational attainment level of the participants was higher than expected. As seen in the table some women chose not to disclose demographic details whilst FMC7, FMC8 and FMC9 joined the groups but did not contribute.

Table 3 - Facemums demographics

The core theme which emerged related to 'Continuity' suggesting both relational and informational continuity can be achieved, using social media as the vehicle to stimulate and engage some women in their maternal health care. Online data from Facebook illustrated the development and maintenance of relational continuity, but concepts of continuity were not discussed between the members, thus the data has not been analysed in further detail here. The interview and focus group data highlights both relational and informational continuity. Management continuity which relates to the communication between clinical care giver was not evident. However there were examples of the Facewives advising the Facemums how to improve management continuity, for example by suggesting the involvement of Consultant Midwives or Supervisors of Midwives in discussions about care planning. Relational continuity was evident for both the participants and the midwife moderators; informational continuity was described by the participants. Verbatim quotations illustrate both informational and relational continuity. Due to the nature of the concepts some quotations demonstrate overlap. The quotations from the participants and midwife moderators are presented in italics. Each participant is anonymised and referred to using the one of the codes from FMB1 to FMB18 and FMC1 - FMC1FMC14. The midwife moderators are FWB1, FWB2, FWC1 and FWC2.

\section{Informational Continuity}

The participants identified that their primary motive for joining the group was to have online contact with a midwife for timely access to information;

FMB12 - What works for me being a first time mum is that any worries or concerns that pop into my head that I feel are not significant enough for me to ring up the community office over the facewives are always at hand to answer! I love that I have that security that if I feel I can't ask anybody else that they are just a few minutes away on my mobile phone! Okay it may not be a reply immediately but that's never an issue as they always have helpful and reassuring information and it is clear they are passionate about their job and looking after us! Makes me feel very safe.'

The Facewives were the primary source of information provision for the women. The Facewives were chosen in preference to the participants named NHS midwife. 
FMB 6 - 'For me it was definitely the facewives for the whole of the pregnancy and the early period when she was born... if I hadn't been able to ask the Facewives I wouldn't really have had anyone to ask.'

However although being able to access a midwife electronically was the principal reason for participating in the study, the speed of the response from the midwife did not appear to be particularly important. Simply being able to ask the question, when it arose was helpful irrespective of the response time,

FMB1 - '...even if the facewives don't answer ...you know that either FWB1 or FWB2 will get back to you.'

FMB12 - '... it may not be a reply immediately but that's never an issue as they always have helpful and reassuring information...'

The women were confident that the Facewives would ensure that the information they provided was accurate and would answer their specific, personal queries;

FMB7- 'I know I have come away from appointments and not asked things that I wanted to know and I would come back from the appointment and post on there and know that FWB1 \&FWB2 even if they didn't know the answer would go away and look it up and get back to me.'

Not only did the women have confidence that the midwives would respond but they felt that the response would better responses they could expect from their NHS named midwife or midwifery team.

FMB7 - I was feeling quite anxious it seemed quite a good way of getting consistent support, but if anything it's been a little bit better, it's been better than I thought...your midwife, you don't really see them that often and when you do it's just a quick five-minute...yeah let's take your bloods... I trust FWB1 and FWB2 a bit more to get a really sound answer, more so than somebody who is rushing a clinic.'

The women were aware of heavy midwifery workloads and tried not make demands on the NHS midwives

FMB18 - 'I would ring my husband and go, 'Right, l'm just going in for my appointment now', and he'd say, 'Right, have you got the things you need to ask', and l'd be like, 'Yeah, I know what I'm asking', and I would come out and go, 'I didn't ask this, I didn't ask any of them'. Because you don't, in an appointment atmosphere, you don't, and I'm always. 'I don't want to take up their time', because my midwife was always half an hour late for everything, and I didn't ever want to...I knew she was already behind.'

FMB7 - 'I've felt I have known much more what is available support-wise. I mean you get leaflets and stuff from your midwives but you are in and out of those appointments so quickly because they are so busy, they have so many women to see, you just don't have time.' 
Informational continuity appears to have been maximised by relational continuity. The women reported having more confidence in the information received because they knew and trusted the midwife imparting it;

FMC 1 - I can ask FWC1 and FWC2 questions, I feel like I know them, you know because you feel that you know them that makes a big difference to me because I feel I can know them so I feel like I can trust them.'

\section{Relational continuity}

A strong midwife-mother relationship was evident and was bi-directional.

FMB1- '... I felt like I knew you when I met you even though l'd never seen you, I felt I knew you, because you know little things...'

FWB1 - 'But I think more than anything, I think when people ask me at work how is it going, how is it, it's the fact that I feel like I know you, I've got to know the women I work with and that has been the best bit.'

Most of the participants reported having a stronger relationship with the Facewives than with their own NHS named midwife;

FMB10 - l'd go to them, l'd never go to my own midwife, l've never phoned her, she was ok but ... I just didn't feel I could pick up the phone to her.'

FMB1 - 'I don't feel I know my own midwife, I don't have the same relationship with my own midwife as I have with FWB1 \& FWB2 and because I do, I feel I know them, it's just different, you feel like you know them more.'

FMB5 - 'My first pregnancy wasn't enjoyable , I had a terrible journey and labour. I feel I wasn't looked after by my midwife, so this time round I feel like I need them more as I am frightened. I feel I have that support now and it makes my whole experience a lot better. I feel I know FWB1 \& FWB2 better than my own midwife. Even though can I just mention my own midwife is great now'.

Some mothers did not know who their named midwife was; in contrast despite not having met the Facewife in person, they felt they knew her.

FMB16 - 'well I never have the same one, I never saw the same community midwives anyway, so I knew more about FWB1 and FWB2 before anyway, and then like when I went into hospital there was FWB2 and she was like 'oooh youre a Facemum aren't you?' And she was like lovely, it was so nice because it was somebody I knew.'

Moreover the lack of face to face contact did not affect the perception of having a relationship. 
FMC3 - 'Even though I didn't know FWC1, obviously, I felt like I knew her because I knew from the forum. That was probably priceless to me really, you couldn't really have written that really.'

Using the social media platform aided continuity in itself by removing some of the barriers to high quality interactions. The site was private and the woman felt safe asking and sharing things they did not want to ask or share elsewhere.

FMB10 - '...I asked about headaches and I would have felt a bit stupid asking triage about that, but I asked on here and facewives came straight on and send me some links and the reply was pretty instant.'

Many participants described feeling that they were being silly or unreasonable seeking advice from their health care providers. Several participants said they felt they were 'mithering'.

FMB3 - 'I do feel silly ringing up, I don't know I do, and if I ask on there (Facemums site) I don't know, I feel better about ringing up, I don't know I always just feel silly just feel I can being a drama queen or wasting time'

But the participants did not feel this way when contacting the Facewives. Furthermore, being able to engage with the Facewives gave the women confidence to access care when they needed it.

FMB3 - 'if the Facewives said to me you need to go to triage or ring them, l'd be like right I will, because sometimes I felt like I was just ringing them pointlessly, so l'd like listen to the Facewives, if the midwife says you need to ring them you know you can do it.'

The ability of the Facewives being able to choose when they responded was important to the participants because they did not feel they were adding to acute pressure;

FMB7 - 'you see the pressure, you see their diaries (midwives) you know how much pressure they're under so to start asking questions ...so I can text and ask questions but equally you get some input from the group which is quite good rather than just... You know how busy midwives are; you know how much they've got to do whereas if people have got access to this kind of thing ...it takes a bit of pressure off them.'

When conventional midwifery care lapsed because of a perceived greater need for obstetric care and involvement, the Facewives were able to bridge gaps to create and maintain midwifery input,

FMB2 - I didn't have a midwife, when I went for diabetic checks there was a midwife but really I just saw the consultant. These (the Facewives) were my midwife.'

Continuity of care is not only important for women, it is an important factor in job satisfaction for midwives (Sandall et al., 2016) .The midwife moderators reported that 
the continuity enabled them to see the participants as women with lives and not just patients to be seen;

FWB2 - I think it's kept me on a level ground really, because you got that little view into each woman's life and you have a better understanding of what these women are going through and you see everything that they've got going on... It is easier on the ward when you got these tasks... And a woman asks you for a water jug a new kind of thing arghhh... It opened your eyes a little bit to people's lives and how they see the systems because as midwives we don't usually get to see that, you might get one person's feedback you know the kind of 'oh she was really snippy with me on triage' but in general you don't get that much feedback about the service, whereas on this we did every single step of the way really.'

FWC2 - You get to know the women, so it wasn't just your straightforward relationship and you know, it just keeps... coming back to...continuity.'

FWC1 -'I just think for me, the best thing, I just felt a bit more like a midwife. That's genuinely the best thing that I found. It was more, like what you thought being a midwife was going to be. I don't get to know the women that I look after. I think my last shift I did four deliveries in 10 hours, so I did not know them...I can honestly say I didn't know any of those four women, so I think that was the best for me really, because sometimes you are so removed from being an actual midwife.'

The Facewives enjoyed being able to provide midwifery support via the Facemums site,

FWC2 - 'I've thoroughly enjoyed it. I think it's been absolutely ground breaking really, in some senses and just like the women, I think about how lucky l've been to be part of it.'

FWB1 - 'It's the best thing l've ever done, in terms of midwifery. Definitely, it was just...it was a lot more than I expected. Well, I don't even know really what we were expecting.'

FWB2- 'it just surprised me how good it was really, it was good because it was just beat my expectations from what I had in the beginning.'

The Facewives felt they had more control over their working lives and the relationships they were able to develop with women;

FWB1 - 'it was just brilliant. You've got work going on and I think the thing when you work for the NHS is that there's a massive institution, you're dealing with lots of different problems, but this was something I felt I had control over, and I felt I had access genuinely to women and their lives and caring for them... I wanted that opportunity to create relationships, and that's what the site's given me.'

\section{Discussion}


The women joined this study to have electronic access to midwives; they wanted to be able to access information and professional advice when they needed it. They were seeking informational continuity i.e. timely access to relevant information. However, it is apparent that although this was their main motive for participating in the study, when they knew that a midwife would respond to their queries, the speed of the response became less important to them. The act of posting a query online appeared to offload some of the anxiety associated with the query. The participants were confident that the Facewives would reply and that in itself reassured them. Peer-support was provided by other Facemums and this advice and information was verified later by the Facewives.

The women in the study expressed feeling foolish and unreasonable when they sought advice from NHS midwives during unscheduled contacts, but did not experience this when contacting the Facewives. Furthermore the online nature of the communication made it easier for some of the mothers to seek advice. Although they were not anonymous within the group, Facemums was situated outside the members usual social environments possibly aiding more open information seeking and sharing (Hasler, Ruthven \& Buchanan, 2014). Participants felt reassured that they weren't adding to the midwives already heavy workload, there was an understanding that the midwives would answer when it was convenient for them to do so. This understanding meant that the women asked when they needed to do so without hesitation, because they didn't feel that they were being burdensome or overdemanding. Previous research has found an association with professional online support and reduced emergency room admissions and there are suggestions that online support can result in more effective use of healthcare services (Nolan et al., 2017; Hudson et al., 2012; Dunham et al., 1998). Professional midwifery advice given in the context of a relationship based forum may have the potential to reassure mothers so that do not feel they need to call and attend largely oversubscribed secondary care settings. Moreover the quality of the interactions and information provided may be improved as women do not feel the same sense of urgency during online communications that they feel during face to face clinical care episodes.

The lack of face to face interaction within a virtual community did not negatively affect the development of a relationship between the midwives and the mothers. Strong relationships formed and relational continuity was achieved in the virtual meeting space. Relational continuity is important for a positive childbearing experience, and the quality of the relationship has the potential to promote positivity throughout the childbirth continuum (Sandall et al., 2016; Sandall et al., 2016a; Dahlberg \& Aune, 2013). This study has demonstrated that relationships between mothers and midwives are not necessarily determined by, or dependent on face to face personal interactions. A high level of relational satisfaction can be achieved through regular online contact. Additionally it appears that interactions do not need to be specific or personal to the individual, but a sense of being available if and when required is important.

The midwife moderators in this study did not physically meet all of the participants, although they met some of them at the focus groups, but despite this they were able to provide a sense of midwifery continuity which was valued by the participants. Accessing professional midwifery advice and support electronically met both the 
informational and relational needs of the participants and in many of the women's experiences the online midwifery support surpassed the care and service provided by the local NHS midwives.

Models of care that allow midwives to develop relationships with women and to provide continuity of care are associated with increased job satisfaction (Newton, McLachlan, Willis \& Forster, 2014; Warmelink et al., 2015; Kirkham et al., 2006). This study was welcomed by participant midwives who expressed satisfaction both in terms of their online contact with mothers and the quality of their midwife/mother relationships. Moderating the group was viewed positively by the midwives who expressed increased job satisfaction as a result. However work life balance for midwives providing continuity is linked with occupational burnout and stress (Yoshida \& Sandall, 2013). Whilst these phenomena were not observed in this study this may not be the case if the model was ongoing, particularly if midwives treated the model as a social interaction rather than a job. The midwives recruited for this study may have put themselves forward because of their positive bias to social media. Nonetheless in this study the ability to provide continuity was a liberating and not restricting aspect of the role for the Facewives.

Controversy surrounds continuity models of care as the standard model for high quality midwifery care. Much of the controversy focuses on the potential expense, both financial and human, of such a model (NHS England, 2016; Sandall et al., 2016a). Barriers are presented which suggest continuity is not a feasible at scale, and models of care which do not have relational continuity at their core continue to be the norm are maintained (NHS England, 2016). The option for women to join a midwife moderated network such as that described could facilitate information and relational continuity via online relationships, thus producing high quality midwifery care, increased satisfaction for mothers, with flexible working for midwives and at less financial expense.

There are potential advantages as well as disadvantages to Facebook continuity models which should be considered.

Table 4 - Positive and negative features of a Facebook continuity model for mothers and midwives

\section{Limitations}

In line with the qualitative approach taken, this study was small, the participants selfselecting and the midwives were essentially self-selecting too as they were keen to engage in a social media based project. The midwives were all based in secondary care thus limiting their opportunities to create and maintain professional midwife mother relationships. Further work is required to understand the elements of this model that impacted on continuity and whether they could be generalized and introduced at scale. Nevertheless these initial findings are sufficiently encouraging to warrant a larger cohort study in the near future.

\section{Conclusion}

Professionally moderated social media based groups can meet information and relational continuity needs of women. They may provide an alternative means of facilitating the continuity that is so often lacking in traditional models of care. 


\section{References}

Bacigalupe, G. (2011). Is There a Role for Social Technologies in CollaborativeHealthcare? Families, Systems, \& Health, 29(1), 1-14.

Braun, V., \& Clarke, V. (2013). Successful qualitative research: A practical guide for beginners. London Sage.

Council, N. a. M. (2008). The code: Standards of conduct, performance and ethics for nurses and midwives NMC: London.

Dahlberg, U., \& Aune, I. (2013). The woman's birth experience-The effect of interpersonal relationships and continuity of care. Midwifery, 29(4), 407-415.

Darvill, R., Skirton, H., \& Farrand, P. (2010). Psychological factors that impact on women's experiences of first-time motherhood: a qualitative study of the transition. Midwifery, 26(3), 357-366. doi: http://dx.doi.org/10.1016/j.midw.2008.07.006

DB, H., C, C.-G., \& M, H. (2012). Effects of an internet intervention on mothers' psychological, parenting, and health care utilization outcomes. Issues in Comprehensive Pediatric Nursing 35, 176-193.

DH. (1993). Changing Childbirth: Part 1: Report of the Expert Maternity Group. London: HMSO.

DH. (2007). Maternity Matters: Choice, access and continuity

of care in a safe service. London: HMSO.

DH. (2010). Midwifery 2020: Delivering expectations London: HMSO.

Duggan, M., \& Smith, A. (2013). Social Media Update 2013. Retrieved August 2015, 2015, from http://www.pewinternet.org/2013/12/30/social-media-update$\underline{2013 /}$

Dunham, P. J., Hurshman, A., Litwin, E., Gusella, J., Ellsworth, C., \& Dodd, P. W. (1998). Computer-mediated social support: single young mothers as a model system. American Journal of Community Psychology, 26(2), 281-306.

Fox, S. (2011). The Social life of Health Information. Pew Internet and American Life project. Retrieved 26 OCtober, 2016, from http://www.pewinternet.org/2011/05/12/the-social-life-of-health-information$2011 /$

Freeman, G. K., Woloshynowych, M., Baker, R., Boulton, M., Guthrie, B., Car, J., . . . Tarrant, C. (2007). Continuity of care 2006: what have we learned since 2000 and what are policy imperatives now. London: National Coordinating Centre for Service Delivery and Organisation.

Guthrie, B., Saultz, J. W., Freeman, G. K., \& Haggerty, J. L. (2008). Continuity of care matters. British Medical Journal (BMJ), 337, a867.

Hasler, L., Ruthven, I., Buchanan, S. (2014) Using internet groups in situations of information poverty: Topics and information needs. Journal of the Association for Information Science and Technology. 65 (1) pp25-36

Heaton, J., Corden, A., \& Parker, G. (2012). Continuity of care': a critical interpretive synthesis of how the concept was elaborated by a national research programme. International Journal of Integrated Care [serial online], 12.

Hudson, D.B., Campbell-Grossman, C. and Hertzog, M., 2012. Effects of an internet intervention on mothers' psychological, parenting, and health care utilization outcomes. Issues in comprehensive pediatric nursing, 35(3-4), pp.176-193.

Kenny, C., Devane, D., Normand, C., Clarke, M., Howard, A., \& Begley, C. (2015). A cost comparison of midwife-led compared with consultant-led maternity care in Ireland (The MidU Study) Midwifery, 31(11), 1032-1038. doi:

doi:10.1016/j.midw.2015.06.012 
Kirkham, M., Morgan, R.K. and Davies, C., 2006. Why Do Midwives Stay? Women's Informed Childbearing and Health Research Group. University of Sheffield, Sheffield.

Marmot, M. G., Allen, J., Goldblatt, P., Boyce, T., McNeish, D., Grady, M., \& Geddes, I. (2010). Fair society, healthy lives: Strategic review of health inequalities in England post-2010.

McLachlan, H. L., Forster, D. A., Davey, M. A., Farrell, T., Flood, M., Shafiei, T., \& Waldenström, U. (2016). The effect of primary midwife-led care on women's experience of childbirth: results from the COSMOS randomised controlled trial. BJOG: An International Journal of Obstetrics \& Gynaecology, 123(3), 465-474. doi: 10.1111/1471-0528.13713

Newton, M. S., McLachlan, H. L., Willis, K. F., \& Forster, D. A. (2014). Comparing satisfaction and burnout between caseload and standard care midwives: findings from two cross-sectional surveys conducted in Victoria, Australia. BMC Pregnancy and Childbirth, 14(1), 426.

NHS England. (2016). National Maternity Review. Better Births; Improving Outcomes of Maternity Services in England. NHS England, London.

Nolan, S., Hendricks, J., Ferguson, S., \& Towell, A. Social networking site (SNS) use by adolescent mothers: Can social support and social capital be enhanced by online social networks? - A structured review of the literature. Midwifery. doi: http://dx.doi.org/10.1016/j.midw.2017.03.002

Nursing and Midwifery Council. (2012). Midwives rules and standards 2012. london: Nursing and Midwifery Council.

Nursing and Midwifery Council. (2015). Guidance on using social media responsibly. London NMC.

Page, L., \& McCandlish, R. E. n. E. N. (2006). The New Midwifery. Science and Sensitivity in Practice (2nd Edition ed.). Netherlands Churchill Livingstone Elsevier.

Renfrew, M. J., McFadden, A., Bastos, M. H., Campbell, J., Channon, A. A., Cheung, N. F., Declercq, E. (2014). Midwifery and quality care: findings from a new evidence-informed framework for maternal and newborn care. Lancet, 384(9948), 1129-1145. doi: 10.1016/s0140-6736(14)60789-3

Sandall, J., Soltani, H., Gates, S., Shennan, A., \& Devane, D. (2016). Midwife-led continuity models versus other models of care for childbearing women. The Cochrane Library.

Sandall, J., Coxon, K., Mackintosh, N., Rayment-Jones, H., Locock, L. and Page, L. (2016a) (writing on behalf of the Sheila Kitzinger symposium) Relationships: the pathway to safe, high-quality maternity care. Report from the Sheila Kitzinger symposium at Green Templeton College October 2015. Green Templeton College, Oxford.

Shribman, S., \& Billingham, K. (2009). Healthy Child Programme - Pregnancy and the first five years Department of Health Best Practice Guidance.

Tracy, S.K., Hartz, D.L., Tracy, M.B., Allen, J., Forti, A., Hall, B., White, J., Lainchbury, A., Stapleton, H.,Beckmann, M. and Bisits, A. (2013). Caseload midwifery care versus standard maternity care for women of any risk: M@ NGO, a randomised controlled trial. The Lancet, 382(9906), pp.17231732.

Tracy, S., Welsh, A., Hall, B., Hartz, D., Lainchbury, A., Bisits, A., \& Tracy, M. (2014). Caseload midwifery compared to standard or private obstetric care for first time mothers in a public teaching hospital in Australia: a cross sectional 
study of cost and birth outcomes. BMC Pregnancy and Childbirth, 14(46). doi: http://doi.org/10.1186/1471-2393-14-46

Vasilica, CM (2015), Impact of using social media to increase patient information provision, networking and communication, PhD thesis, University of Salford. Available from http://usir.salford.ac.uk/view/authors/58242.html

Walsh, D., \& Devane, D. (2012). A Metasynthesis of Midwife-Led Care. Qual Health Res, 22(7), 897-910. doi: 10.1177/1049732312440330

Warmelink, J. C., Hoijtink, K., Noppers, M., Wiegers, T. A., de Cock, T. P., Klomp, T., \& Hutton, E. K. (2015). An explorative study of factors contributing to the job satisfaction of primary care midwives. Midwifery, 31(4), 482-488. doi: 10.1016/j.midw.2014.12.003

Yoshida, Y., \& Sandall, J. (2013). Occupational burnout and work factors in community and hospital midwives: a survey analysis. Midwifery, 29(8), 921926. doi: 10.1016/j.midw.2012.11.002 